Bundesgesundheitsbl 2019 · 62:1140-1143 https://doi.org/10.1007/s00103-019-02989-9 c) Springer-Verlag GmbH Deutschland, ein Teil von Springer Nature 2019

Mitteilungen des Arbeitskreises Blut des Bundesministeriums für Gesundheit

\title{
Stellungnahme Fehlanwendungen von Blutkomponenten
}

\section{Bei der 87. Sitzung des Arbeitskreises Blut am 14.05.2019 wurde folgende Stellungnahme (S 19) verabschiedet}

\section{Ausgangssituation}

Sicherheit und Qualität von Blutprodukten konnten durch Verbesserungen bei der Auswahl der spendenden Personen, der Spendertestung sowie den Herstellungs- und Lagerprozessen in den letzten Jahren deutlich verbessert werden. Der Qualitätsstandard der Blutprodukte in Deutschland ist so hoch wie nie zuvor.

Neben der Produktqualität und -sicherheit und der richtigen Indikationsstellung kommt in der Hämotherapie der Prozesssicherheit eine erhebliche Bedeutung zu. Hierbei spielt die Vermeidung von Fehltransfusionen eine zentrale Rolle. Diagnostische Maßnahmen und vor allem die Identitätssicherung bzw. die korrekte Zuordnung ausgewählter Blutprodukte zu einer bestimmten Patientin bzw. einem bestimmten Patienten sind hierbei von großer Sicherheitsrelevanz.

Trotz aller bereits bestehenden Qualitätssicherungsschritte weisen Hämovigilanzberichte und Publikationen sowie klinische Erfahrungen darauf hin, dass „Fehltransfusionen“ ein signifikantes Risiko im Prozess der transfusionsmedizinischen Versorgung darstellen.

Fehltransfusionen werden im Hämovigilanzbericht des Paul-Ehrlich-Instituts wie folgt definiert: „Fehltransfusionen sind Transfusionen, bei denen die zu transfundierenden Blutkomponenten dem falschen Patienten zugeordnet oder verabreicht wurden, was meist eine Transfusion von Komponenten mit nicht identischer Blutgruppe zur Folge hat. $\mathrm{Zu}$ den Fehltransfusionen gehören aber auch z. B. die Gabe unbestrahlter Blutkomponenten trotz entsprechender Anforderung oder die blutgruppenkompatible Transfusion bei Patienten ohne Transfusionsindikation." [1].

Im SHOT-Bericht („Serious Hazards of Transfusion Report“) werden unter Fehltransfusionen (,incorrect blood component transfused“) zusätzlich auch zu spät oder nicht erfolgte Bluttransfusionen erfasst [2].
In den Kalenderjahren 2015 bis 2017 wurden dem Paul-Ehrlich-Institut insgesamt 209 Fehltransfusionen von Erythrozytenkonzentraten gemeldet ( $\bullet$ Tab. 1). In 79 Fällen traten Transfusionsreaktionen auf, davon nahmen sechs einen tödlichen Verlauf. Die Häufigkeit von gemeldeten Fehltransfusionen insgesamt lag bei 19,3 bestätigten Fällen pro Million transfundierter Erythrozytenkonzentrate.

Im Rahmen des SHOT-Berichts 2017 wurde aus Großbritannien berichtet, dass 2016 und 2017 insgesamt 4 Fehltransfusionen und 606 sogenannte Beinahe-Fehltransfusionen von Erythrozytenkonzentraten gemeldet wurden.

Die häufigste Fehlerquelle bei diesen Vorfällen waren falsche Blutgruppenzuordnungen zur Patientin bzw. zum Patienten (falsches Blut im Röhrchen= „wrong blood in tube " $=$ WBIT) und nicht oder falsch durchgeführte patientennahe Kontrollen. Im Jahre 2017 konnten 317 von 342 Beinahe-Fehltransfusionen auf WBIT zurückgeführt werden [2]. Dies zeigt, dass

Tab. 1 Bestätigte Fehltransfusionen von Erythrozytenkonzentraten mit und ohne Transfusionsreaktion. Adaptiert aus dem: HämovigilanzBericht des Paul-Ehrlich-Instituts 2016/17

\begin{tabular}{|c|c|c|c|c|}
\hline Fehltransfusionen & 2015 & 2016 & 2017 & 2015-2017 \\
\hline $\begin{array}{l}\text { Fehltransfusionen mit Transfusionsreaktion } \\
\text { (gesamt) }\end{array}$ & 24 & 28 & 27 & 79 \\
\hline Fehltransfusionen mit tödlichem Verlauf & 3 & 2 & 1 & 6 \\
\hline Fehltransfusionen ohne Transfusionsreaktion ${ }^{a}$ & 34 & 41 & 55 & 130 \\
\hline Summe der Fehltransfusionen & 58 & 69 & 82 & 209 \\
\hline Verbrauch Erythrozytenkonzentrate & 3.754 .760 & 3.548 .124 & 3.506 .417 & 10.809 .301 \\
\hline
\end{tabular}


die identitätsgesicherte Blutabnahme bei der zur behandelnden Person der wesentliche Schritt ist.

Betrachtet man die internationale Literatur, so ergibt sich eine durchschnittliche Häufigkeit für WBIT von circa 1 bei 2000 Blutentnahmen [3, 4]

\section{Empfehlungen zur Vermeidung von Fehlanwendungen von Blutkomponenten}

Um den Gesamtprozess der Hämotherapie und der transfusionsmedizinischen Behandlung weiter $\mathrm{zu}$ verbessern und noch sicherer zu machen, sollten eine Reihe von zusätzlichen Maßnahmen eingeführt werden, die dazu beitragen, die Zahl von Fehltransfusionen weiter zu reduzieren.

\section{Qualifikation der Blutkomponenten anwendenden Personen}

\subsection{Aufnahme der Hämotherapie in das Curriculum des Medizinstudiums}

Die medizinischen Fakultäten der Universitäten und Hochschulen werden dringend ersucht, die Hämotherapie mit theoretischer Ausbildung und praktischen Übungen verpflichtend in das Curriculum des Medizinstudiums aufzunehmen.

\subsection{Einweisung und Schulung aller an der Hämotherapie beteiligten Ärztinnen und Ärzte}

Die in der Hämotherapierichtlinie [5] vorgesehene Einweisung transfundierender Ärztinnen und Ärzte durch die transfusionsbeauftragte Person muss insbesondere Informationen zur sicheren Anwendung und die möglichen Konsequenzen einer Fehlanwendung sowie deren Behandlung und Maßnahmen zur Vermeidung unerwünschter Ereignisse, z.B. WBIT, Fehltransfusionen und „near misses“, beinhalten. In dieser Einweisung muss auch über die Meldeverpflichtung bei Fehltransfusionen und Transfusionsreaktionen informiert werden.

Diese Inhalte sollen in jährlichen Schulungen aller transfundierenden Ärztinnen und Ärzte durch die transfusionsbeauftragte Person aktualisiert werden.

Die Einrichtung der Krankenversorgung hat sicherzustellen, dass alle transfusionsbeauftragten Personen mindestens alle fünf Jahre geschult werden, um sie zu befähigen, die obigen Inhalte zu vermitteln.

\subsection{Erstellung von (Muster)- Schulungsmaterialien}

Die Deutsche Gesellschaft für Transfusionsmedizin und Immunhämatologie (DGTI) wird gebeten, (Muster)-Schulungsmaterialien zur Vermeidung von Fehltransfusionen, zur Meldepflicht und zur Ursachenanalyse bei Fehltransfusionen oder Beinahe-Fehltransfusionen zu erarbeiten und dem AK Blut zur Verfügung zu stellen.

\section{Optimierung der Umsetzung bisheriger Meldepflichten}

Zur Risikoeinschätzung von Fehltransfusionen kommt der Erhebung und Auswertung transfusionsepidemiologischer Daten eine große Bedeutung zu. Aktuell bestehen regulatorische Vorgaben zu Dokumentations-, Melde- und Unterrichtungspflichten nach Arzneimittel- und Transfusionsgesetz. Die Meldepflichten sind in der Richtlinie Hämotherapie [5] im Einzelnen festgelegt.

Hierzu empfiehlt der Arbeitskreis Blut folgende Maßnahmen:

\subsection{Information an alle Krankenhäuser}

Die Deutsche Krankenhausgesellschaft wird gebeten, in regelmäßigen Abständen alle Krankenhäuser auf die Meldepflichten für alle in die Transfusion von Blutkomponenten involvierten Mitarbeiterinnen und Mitarbeiter hinzuweisen.

\subsection{Information aller Ärztinnen und Ärzte}

Die Landesärztekammern werden gebeten, Ihre Mitglieder in regelmäßigen Abständen auf die o.g. Meldepflichten hinzuweisen.

\section{Meldeverpflichtung für „near misses" („Beinahe-Schäden“) an den Qualitätsbeauftragten für Hämotherapie (QBH) und Integration der Fehlermeldungen einschließlich „Beinahe-Schäden" in das Qualitätssicherungssystem der Einrichtung}

Wird eine Fehltransfusion bekannt, die für die Blutprodukte empfangende Person folgenlos, d.h. ohne transfusionsmedizinische Reaktion oder klinischen Schaden abläuft, besteht dennoch für die Zulassungsinhaberin oder den Zulassungsinhaber die Verpflichtung, dies als schwerwiegenden Zwischenfall der Bundesoberbehörde zu melden ( $\$ 63$ i Abs. 6 Arzneimittelgesetz). Für die behandelnde ärztliche Person ergibt sich bei einer fehlerhaften Anwendung derzeitig keine regulatorische Verpflichtung, dies dem Paul-Ehrlich-Institut zu melden. Das Ereignis muss jedoch von der behandelnden ärztlichen Person der transfusionsbeauftragten Person und der transfusionsverantwortlichen Person gemeldet oder an die im Qualitätssicherungssystem definierte Stelle gemeldet werden. $(\$ 16 \mathrm{Ab}$ satz 1 Transfusionsgesetz). Hierzu gehören auch die „near misses“.

Unter Verweis auf die Definitionen des Ärztlichen Zentrums für Qualität in der Medizin wird der englischsprachige Begriff „, near misses“ im Folgenden mit „Beinahe-Schaden “ übersetzt und als „ein Fehler ohne Schaden, der zu einem Schaden hätte führen können" definiert [6].

Beinahe-Schäden sind zum Beispiel: Verwechslungen bei der Blutabnahme („wrong blood in tube“, WBIT), Fehltransfusionen ohne klinische Konsequenz sowie durch einen Bed-Side-Test bzw. die Identitätskontrolle und Produktüberprüfung noch verhinderte Fehltransfusionen.

Im Qualitätssicherungs-System der Einrichtung sind unter Beachtung von Kapitel 6.4 der Hämotherapierichtlinien [5] Anweisungen zum Umgang mit Fehlern und zur Vermeidung von Fehlern zu erstellen:

- Maßnahmen zur Gefahrenabwehr Bei der Entdeckung eines Fehlers oder „near miss“ sind unverzüglich notwendige Maßnahmen zur Gefah- 
renabwehr einzuleiten (z.B. WBIT: Ausschluss einer Verwechslung und ggf. Identifizierung weiterer betroffener Patientinnen und Patienten)

- Meldung an die transfusionsbeauftragte Person und die transfusionsverantwortliche Person

- Fehlerursachenanalyse durch die transfusionsbeauftragte Person

- Bewertung durch die transfusionsbeauftragte Person

- Bericht inklusive Maßnahmenempfehlung durch die transfusionsbeauftragte Person an die transfusionsverantwortliche Person

Die transfusionsverantwortliche Person erstellt eine Zusammenfassung der Einzelberichte als jährlichen Bericht an die Transfusionskommission.

Die Transfusionskommission bewertet diesen Bericht und entwickelt auf Basis dieser Daten eine Maßnahmenempfehlung für die Leitung der Einrichtung. Im Rahmen der Transfusionskommission wird die Umsetzung der Maßnahmen zur Erhöhung der Transfusionssicherheit regelmäßig evaluiert.

\section{Sicherheits-Checkliste für die Transfusion von Erythrozytenkonzentraten}

Die Prozesssicherheit der Transfusion von Blutprodukten, insbesondere von Erythrozytenkonzentraten, ist an eine Reihe notwendiger Schritte gebunden. Die Nichterfüllung jedes einzelnen Schritts kann als Konsequenz eine Fehltransfusion nach sich ziehen. Deshalb ist in Anlehnung an Qualitätssicherungsmaßnahmen in anderen medizinischen Bereichen dringend die Einführung einer - auch elektronischen - „Sicherheits-Checkliste“ für jede Transfusion einer Blutkomponente zu empfehlen, sofern die klinische Situation dies zulässt.

Diese soll mindestens die Indikationsstellung, die positive Identifizierung der Patientin bzw. des Patienten, die Kompatibilität der Blutgruppe mittels „BedSide-Test“, die Überprüfung des Blutpräparates bezüglich Übereinstimmung zwischen Chargennummer der Blutkomponente und Begleitschein, die Überprüfung der Gültigkeit der immunhämatolo- gischen Verträglichkeitsprobe sowie die Überprüfung des Haltbarkeitsdatums der Blutkomponente beinhalten. Hinweise zur Umsetzung finden sich in der Muster-Arbeitsanweisung zur Transfusion von Erythrozytenkonzentraten (EK) der Bundesärztekammer [7].

\section{IT-gestützte Identitätssicherung}

Im Gesamtprozess der Blutkomponententransfusion ist eine Vielzahl von Prozessrisiken im Sinne von Verwechslungen möglich und realistisch. Dieses beginnt bei der Indikationsstellung zur Transfusion, führt über „Wrong Blood in Tube“ und Laborfehler bis hin zur möglichen Transfusion eines Produktes an eine "falsche“ Patientin oder einen „falschen“ Patienten. Die wissenschaftliche Literatur belegt, dass die Anwendung von IT-gestützten Systemen, z. B. unter Verwendung von Armbändern für behandelte Personen, die Quote von Medikationsfehlern und WBIT signifikant senkt $[8,9]$.

Es ist zu fordern, dass entsprechend geeignete IT-Programme, mit denen ein Abgleich der Barcodes von Blutpräparat und Fallnummer mit der Datenbank des Labors erfolgen kann, entwickelt und eingesetzt werden. Der Einsatz einer IT-gestützte Identitätssicherung sollte im Rahmen einer nationalen Pilotstudie untersucht werden, um den Nutzen dieser Maßnahme exemplarisch belegen zu können.

\section{Rechtliche Vorgaben}

Es wird darauf hingewiesen, dass Verpflichtungen nach dem Arzneimittelgesetz, dem Transfusionsgesetz, der Richtlinie Hämotherapie sowie ggf. sonstige rechtliche Vorgaben durch die vorliegende Stellungnahme unberührt bleiben.

\section{Glossar (Auszüge aus [5], Kapitel 7.1)}

Schwerwiegende Nebenwirkungen sind Nebenwirkungen, die tödlich oder lebensbedrohend sind, eine stationäre Behandlung oder Verlängerung einer stationären Behandlung erforderlich machen, zu bleibender oder schwerwiegender Behinderung, Invalidität, kongenitalen An- omalien oder Geburtsfehlern führen ( $\$ 4$ Abs. 13 S. 3 AMG)

Schwerwiegende unerwünschte $\mathrm{Re}$ aktion ist eine unbeabsichtigte Reaktion, einschließlich einer übertragbaren Krankheit, bei der spendenden Person oder der empfangenden Person im Zusammenhang mit der Gewinnung von Gewebe oder Blut oder der Übertragung von Gewebe- oder Blutzubereitungen, die tödlich oder lebensbedrohend verläuft, eine Behinderung oder einen Fähigkeitsverlust zur Folge hat oder einen Krankenhausaufenthalt erforderlich macht oder verlängert oder zu einer Erkrankung führt oder diese verlängert (\$63i Abs. 7 AMG)

Schwerwiegender Zwischenfall ist jedes unerwünschte Ereignis im $\mathrm{Zu}$ sammenhang mit der Gewinnung, Untersuchung, Aufbereitung, Be- oder Verarbeitung, Konservierung, Aufbewahrung oder Abgabe von Geweben oder Blutzubereitungen, das die Übertragung einer ansteckenden Krankheit, den Tod oder einen lebensbedrohenden Zustand, eine Behinderung oder einen Fähigkeitsverlust von Patientinnen oder Patienten zur Folge haben könnte oder einen Krankenhausaufenthalt erforderlich machen oder verlängern könnte oder zu einer Erkrankung führen oder diese verlängern könnte (\$63i Abs. 6 AMG)

Für den Arbeitskreis Blut

\section{Dr. R. Offergeld}

Vorsitzende

Dieses Papier wurde erarbeitet von den Mitgliedern der Untergruppe „Fehltransfusionen" des Arbeitskreises Blut: Prof. Dr. Dr. h.c. Erhard Seifried, Prof. Dr. Markus Funk, Prof. Dr. Birgit Gathof, Dr. Jochen Hoch, Dr. Gabriele Hutschenreuter, Dr. Reinhard Kasper.

\section{Literatur}

1. Funk $M$, Heiden $M$, Müller $S$ et al Hämovigilanzbericht des Paul-Ehrlich-Instituts 2016/2017. www. pei.de/haemovigilanzbericht. Zugegriffen: 17. Juni 2019

2. Bolton-Maggs PHB (Ed) Poles D et al (2018) The 2017 Annual SHOT Report. https://www.shotuk. org/wp-content/uploads/myimages/SHOT-Report-2017-WEB-Final-v4-25-9-18.pdf. Zugegriffen: 17. Juni 2019

3. Bolton-Maggs PH, Wood EM, Wiersum-Osselton JC (2015) Wrong blood in tube - potential for serious 
outcomes: can it be prevented? $\mathrm{Br} J$ Haematology 168(1):3-13

4. Strauss R, Downie H, Wilson A (2018) Sample collection and sample handling errors submitted to the transfusion error surveillance system, 2006 to 2015. Transfusion 58(7):1697-1707

5. Bundesärztekammer (2017) Richtlinie zur Gewinnung von Blut und Blutbestandteilen und zur Anwendung von Blutprodukten (Richtlinie Hämotherapie). https://www.bundesaerztekammer.de/fileadmin/user_upload/downloads/pdfOrdner/MuE/Richtlinie_Haemotherapie_2017.pdf. Zugegriffen: 17. Juni 2019

6. Ärztliches Zentrum für Qualität in der Medizin (2019) Definitionen und Klassifikation zur Patientensicherheit. https://www.aezq.de/patientensicherheit/definition-ps. Zugegriffen: 17. Juni 2019

7. Bundesärztekammer (2019) Muster-Arbeitsanweisung zur Transfusion von Erythrozytenkonzentraten (EK) unter den besonderen Bedingungen des Abschnitts 6.4.2.3.1 b) „Sonderfälle“. https://www. bundesaerztekammer.de/aerzte/medizin-ethik/ wissenschaftlicher-beirat/veroeffentlichungen/ haemotherapie-transfusionsmedizin/. Zugegriffen: 17. Juni 2019 (der Richtlinie zur Gewinnung von Blut und Blutbestandteilen und zur Anwendung von Blutprodukten (Richtlinie Hämotherapie), Gesamtnovelle 2017)

8. Higgins T, Heelon M, Siano B et al (2010) Medication safety improves after implementation of positive patient identification. Appl Clin Inform 1:213-220

9. Kaufman RM, Dinh A, Cohn CS et al (2019) Electronic patient identification for sample labeling reduces wrong blood in tube errors. Transfusion 59:972-980 